\title{
Vaccine hesitancy and COVID-19 vaccination in Hong Kong
}

\author{
Paul KS Chan ${ }^{1}$ *, MD, FRCPath, Martin CS Wong ${ }^{2}$, MD, MPH, Eliza LY Wong ${ }^{2}$, PhD, FHKCHSE \\ ${ }^{1}$ Department of Microbiology, ${ }^{2}$ The Jockey Club School of Public Health and Primary Care, Faculty of Medicine, The Chinese University \\ of Hong Kong, Hong Kong \\ *Corresponding author: paulkschan@cuhk.edu.hk \\ Hong Kong Med J 2021;27:90-1
}

https://doi.org/10.12809/hkmj215115

Although the case fatality rate of coronavirus disease 2019 (COVID-19) is lower compared with deadly diseases such as smallpox and Ebola virus disease, ${ }^{1}$ the associated health and economic burden is alarming. Shifting healthcare technology and facilities to vaccine development and massive production became an international common goal. The arrival of COVID-19 vaccines was perceived as the end of health and economic suffering, and the rebirth of tourism and many other industries. Data from clinical trials of vaccines made by both the new and conventional platforms showed promising results, but the rolling out of vaccination is really challenging in some parts of the world.

In 2019 before the emergence of COVID-19, the World Health Organization (WHO) identified vaccine hesitancy as one of 10 major threats to global health. ${ }^{2}$ Hong Kong has a comprehensive childhood immunisation programme with an excellent uptake, and vaccine hesitancy is not often considered a problem locally. However, there are bits and pieces of information indicating that this may not be the case. In May 2009, when the WHO influenza alert level was raised to Phase 5 signifying that a pandemic was imminent, our survey indicated that only $47.9 \%$ of healthcare workers at public hospital intended to accept the flu $\mathrm{H} 1 \mathrm{~N} 1$ vaccine when available. ${ }^{3}$ The acceptance for $\mathrm{H} 5 \mathrm{~N} 1$ vaccine (another flu with pandemic threat) was even lower (34.8\%). Such low intention to accept turned out to be true when the vaccination programme for pandemic flu H1N1 was initiated in Hong Kong.

In early 2005, Hong Kong faced a heavy flu season due to a new influenza strain (H3N2 Switzerland). The government decided to implement an extra dose of vaccine incorporated with the new strain before the summer peak. Healthcare workers again showed a low (31.8\%) intention to accept. ${ }^{4}$

One may argue that, from these data on flu vaccines, we cannot infer a low acceptance of COVID-19 vaccines, because of the vast difference in health and economic impact. However, our repeated cross-sectional studies on the local working population show that acceptance for COVID-19 vaccines has declined from $44.2 \%$ during the first wave to $34.8 \%$ during the third wave of epidemic. ${ }^{5}$
Similarly low vaccine acceptance rates were revealed by another study which included more elderly participants, who are considered as the priority group for vaccination. ${ }^{6}$ That study also identified specific barriers for COVID-19 vaccines. For instance, $43.4 \%$ of participants expressed lack of confidence on vaccines produced by new platforms, $52.2 \%$ considered the track record of vaccine manufacturers important, and $62.5 \%$ regarded the country of vaccine production could affect their acceptance. These are beyond the key safety and efficacy issues that policy makers are focused on. It is notable that government recommendation was the strongest driver for vaccine acceptance, conferring a 10-times-higher odds of receiving vaccines among the study participants.

One may optimistically assume that these opinions on willingness or intention to accept will change when the public are offered vaccines. Unfortunately, despite a massive government-led vaccination campaign, the uptake after 1 month of availability was only about $6 \%$ of the total population in Hong Kong. Had we underestimated the results of pre-rolling out vaccine acceptance surveys? Had we not proactively addressed vaccine hesitancy? Although there are numerous public education and promotion materials on COVID-19 vaccines being disseminated to the public through various media, combating vaccine hesitancy is another ball game. If we do not develop an effective strategic plan to counter vaccine hesitancy, we will be unable to escape from the COVID-19 pandemic. Efforts to develop and produce vaccines for COVID-19 at unprecedented speed and scales may be in vain.

We believe vaccine hesitancy should be addressed by an organised and concerted effort contributed to by various stakeholders in the community. This effort should include more intensive education, provision of more evidencebased information, and public health interventions to enhance vaccine uptake. ${ }^{7}$ Exemption from travel bans, issuance of vaccination certificates, visitation rights at healthcare facilities, and incentives offered by the commercial sector to the employees are some potential strategies to increase the inoculation rate further, and this requires collaborative initiatives driven by healthcare policies. 


\section{Author contributions}

All authors contributed to the concept and design. PKS Chan drafted the manuscript. MCS Wong and ELY Wong critically reviewed the manuscript. All authors contributed to the editorial, approved the final version for publication, and take responsibility for its accuracy and integrity.

\section{Disclosures}

PKS Chan is a member of the Expert Committee on Clinical Events Assessment Following COVID-19 Immunisation for the Hong Kong SAR Government. Other authors have disclosed no conflicts of interest.

\section{References}

1. Streeck H, Schulte B, Kümmerer BM, et al. Infection fatality rate of SARS-CoV2 in a super-spreading event in Germany. Nat Commun 2020;11:5829.

2. World Health Organization. Ten threats to global health in 2019. Available from: https://www.who.int/news-room/ spotlight/ten-threats-to-global-health-in-2019. Accessed
31 Mar 2021.

3. Chor JS, Ngai KL, Goggins WB, et al. Willingness of Hong Kong healthcare workers to accept pre-pandemic influenza vaccination at different WHO alert levels: two questionnaire surveys. BMJ 2009;339:b3391.

4. Wong MC, Nelson EA, Leung C, et al. Ad hoc influenza vaccination during years of significant antigenic drift in a tropical city with 2 seasonal peaks: a cross-sectional survey among health care practitioners. Medicine (Baltimore) 2016;95:e3359.

5. Wang K, Wong EL, Ho KF, et al. Change of willingness to accept COVID-19 vaccine and reasons of vaccine hesitancy of working people at different waves of local epidemic in Hong Kong, China: repeated cross-sectional surveys. Vaccines (Basel) 2021;9:62.

6. Wong MC, Wong EL, Huang J, et al. Acceptance of the COVID-19 vaccine based on the health belief model: A population-based survey in Hong Kong. Vaccine 2021;39:1148-56.

7. Dror AA, Eisenbach N, Taiber S, et al. Vaccine hesitancy: the next challenge in the fight against COVID-19. Eur J Epidemiol 2020;35:775-9. 